\title{
Downrange manoeuvre and oscillation suppression of a self-regulating centrifugally deployed flexible heat shield using a controlled reaction wheel \\ DOI:
}

10.1016/j.actaastro.2018.12.041

\section{Document Version \\ Accepted author manuscript}

Link to publication record in Manchester Research Explorer

Citation for published version (APA):

Wu, R., Roberts, P. C. E., Soutis, C., \& Diver, C. (2019). Downrange manoeuvre and oscillation suppression of a self-regulating centrifugally deployed flexible heat shield using a controlled reaction wheel. Acta Astronautica. https://doi.org/10.1016/j.actaastro.2018.12.041

\section{Published in:}

Acta Astronautica

\section{Citing this paper}

Please note that where the full-text provided on Manchester Research Explorer is the Author Accepted Manuscript or Proof version this may differ from the final Published version. If citing, it is advised that you check and use the publisher's definitive version.

\section{General rights}

Copyright and moral rights for the publications made accessible in the Research Explorer are retained by the authors and/or other copyright owners and it is a condition of accessing publications that users recognise and abide by the legal requirements associated with these rights.

\section{Takedown policy}

If you believe that this document breaches copyright please refer to the University of Manchester's Takedown Procedures [http://man.ac.uk/04Y6Bo] or contact uml.scholarlycommunications@manchester.ac.uk providing relevant details, so we can investigate your claim.

\section{OPEN ACCESS}




\section{Manuscript Details}

\section{Manuscript number}

Title
AA_2018_1159_R1

Downrange manoeuvre and oscillation suppression of a self-regulating centrifugally deployed flexible heat shield using a controlled reaction wheel

\section{Article type}

Research paper

\section{Abstract}

A recent study has introduced a flexible deployable heat shield that passively deploys and stiffens due to centrifugal forces generated from a self-regulated autorotation. This paper demonstrates that the heat shield is similar to a PI controlled second order nonlinear system, which explains why the deployment is accompanied by a limit cycle structural oscillation that persists throughout a simulated re-entry. The heat shield design offers an unique capability to actively adjust the deployment using conventional attitude control devices. This operation is explored by simulating the re-entry of a CubeSat-sized vehicle equipped with an off-the-shelf reaction wheel controlled by a switching phase shift controller and gain-scheduled controllers. The effects of the control parameters are investigated, and successful oscillation suppression as well as an open-loop downrange manoeuvre of over $300 \mathrm{~km}$ is predicted for re-entry from low earth orbit.

\section{Keywords}

\section{Manuscript category}

Corresponding Author

Corresponding Author's Institution

Order of Authors

Suggested reviewers entry vehicle; deployable aerodynamic decelerator; targeted re-entry; centrifugal deployment; limit cycle oscillation

Space Technology \& Systems Development

Rui Wu

The University of Manchester

Rui Wu, Peter Roberts, Constantinos Soutis, Carl Diver

Juan Cruz, Ian Clark, Robert Braun

\section{Submission Files Included in this PDF}

\section{File Name [File Type]}

Cover_Letter.pdf [Cover Letter]

Response to Reviewers.pdf [Response to Reviewers]

Highlights.docx [Highlights]

PDF_manuscript_v2.pdf [Manuscript File]

\section{Submission Files Not Included in this PDF}

\section{File Name [File Type]}

Latex_source.zip [LaTeX Source File]

To view all the submission files, including those not included in the PDF, click on the manuscript title on your EVISE Homepage, then click 'Download zip file'. 


\section{Cover Letter}

Downrange manoeuvre and oscillation suppression of a self-regulating centrifugally deployed flexible heat shield using a controlled reaction wheel

Rui Wu

Postgraduate Researcher, School of Mechanical, Aerospace and Civil Engineering, The University of Manchester

M13 9PL, UK

07522610811

rui.wu@manchester.ac.uk

23 July 2018

Dear Professor Jeng-Shing Chern:

I am pleased to submit an original research article entitled "Downrange manoeuvre and oscillation suppression of a self-regulating centrifugally deployed flexible heat shield using a controlled reaction wheel" by Rui Wu, Peter Roberts, Constantinos Soutis and Carl Diver for consideration for publication in Acta Astronautica.

We previously reported a new type of deployable aerodynamic decelerator (heat shield) that utilises centrifugal force from autorotation to achieve a self-regulating deployment [1]. In this manuscript, we expanded the understanding about its dynamic behaviours and revealed its unique capability to achieve active deployment control and thus downrange manoeuvre using a single reaction wheel. A limit-cycle structural oscillation, which persists throughout simulated re-entry, is also investigated. According to trajectory simulation of a CubeSat-sized system, an off-the-shelf reaction wheel controlled by an algorithm developed in the present study could achieve a considerable downrange manoeuvrability while suppressing the structural oscillation.

We believe that this manuscript is appropriate for publication by Acta Astronautica because it provides a concise solution to targeted re-entry using only a conventional attitude control device (reaction wheel). The successful oscillation suppression also broadens the application of this deployable decelerator design.

This manuscript has not been published and is not under consideration for publication elsewhere. We have no conflicts of interest to disclose.

Thank you for your consideration!

Sincerely,

Rui Wu

\section{Reference}

[1] R. Wu, P. Roberts, C. Soutis, C. Diver, Acta Astronautica (2018), https://doi.org/10.1016/j.actaastro.2018.06.021 


\section{Response to Reviewers of:}

"Downrange manoeuvre and oscillation suppression of a self-regulating centrifugally deployed flexible heat shield using a controlled reaction wheel". Wu, R., Roberts, P. C. E., Soutis, C., and Diver, C.

\section{Reviewer 1}

The manuscript introduces a concept to control the passive self-deployment of a flexible heat shield using traditional attitude control actuators (i.e., a reaction wheel).

When partially deployed the heat shield's shape and folding pattern generate a torque that induces an autorotation in roll. The centrifugal forces caused by this rotation help deploy the heat shield. As the heat shields deploy, the induced roll torque decreases. Therefore the autorotation in roll is self-regulated by the heat-shield. However it appears that this type of heat shields suffers from two problems: 1) oscillatory deploying-folding motion 2) conning motion appears when attempting to generating lift in order to control downrange trajectory.

The authors show via numerical simulations that for heat-shields mounted on CubeSat-like vehicles a single COTS reaction wheel is enough to control the heat shield's deployment and limit the oscillatory deploying-folding motion. This control also allows to modulate the drag and enables limited control on the downrange trajectory.

The manuscript is well written (see below for additional comments) and appears to be technically sound. The contributions are clearly identified and in my opinion they are sufficient. The assumptions of the analysis are clearly stated and are 
reasonable for a proof-of-concept study. However, the figures could be better presented (in terms of clarity and legibility).

\section{Reply:}

Your comments are greatly appreciated. Please find my specific replies below.

General Comments

- In page 6 (first sentence starting in page 5) it is stated that the deployment angle and spin rate are strongly coupled with nearly no phase difference. I believe this statement has some implicit assumptions. As a second order system the deployment angle presents a lag with respect to the deployment torque M_ $\{F c\}$. So in my opinion it is implicitly assumed that the "shell dynamics" are significantly faster than the spacecraft spinning dynamics. If that is the case please state it explicitly, if not please explain why there is no phase difference.

\section{Reply:}

There is a lag that is very small due to the exact reason you mentioned in the comment. To include this information in the text, the underlined sentence below is added to this paragraph, p5-6:

"... with nearly no phase difference (since the dynamics of the shell deployment is significantly faster than the vehicle spinning dynamics, or in other words, the integral gain is low)."

- It is not clear to me if roll drag is included in the simulations. It seems that if a reaction wheel was used to increase the roll rate above its stable condition to force a higher deployment angle (for downrange control) then the drag (in roll) would need to be compensated by the reaction wheel and thus the wheel will 
eventually saturate. Could the authors clarify this point?

Reply:

The roll drag was included in the simulations, as stated in the paragraph after Eq. 13, p8, where it is referred to as "negative torque".

To further clarify this, the following text is added after Eq. 9, p5:

" $\tau$ is treated as a scalar, which is positive/negative when it accelerates/decelerates the autorotation".

You are right about the momentum saturation. Full saturation is inevitable without the anti-saturation gain control. To emphasize the necessity of de-saturation earlier in the text, the following sentence is added as the first sentence of the paragraph before Eq. 14, p7:

"Momentum saturation is inevitable when a biased control is applied, which requires an anti-saturation control."

Specific Comments

- Fig. 5 would benefit from adding the quantities symbols in the axis \theta and $\backslash \operatorname{dot}\{\backslash$ theta\} (this comment also applies to all other figures). Additionally, I would recommend the authors to eliminate the figure title and include that information in the caption. Also, please explain in details what are the initial conditions. Finally, explain what happens at the point where the heat shield reaches theta $=0$ with $a-500 \mathrm{deg} / \mathrm{s}$ velocity.

\section{Reply:}

The figure is revised to add symbols in axis label, delete the title, and add markers to show initial points and direction. The caption is revised to: "A typical limit cycle oscillation from the simulations, 
generated with the $3 \mathbf{~ k g}$ baseline vehicle design under a flight condition at $30 \mathrm{~km}$ during re-entry."

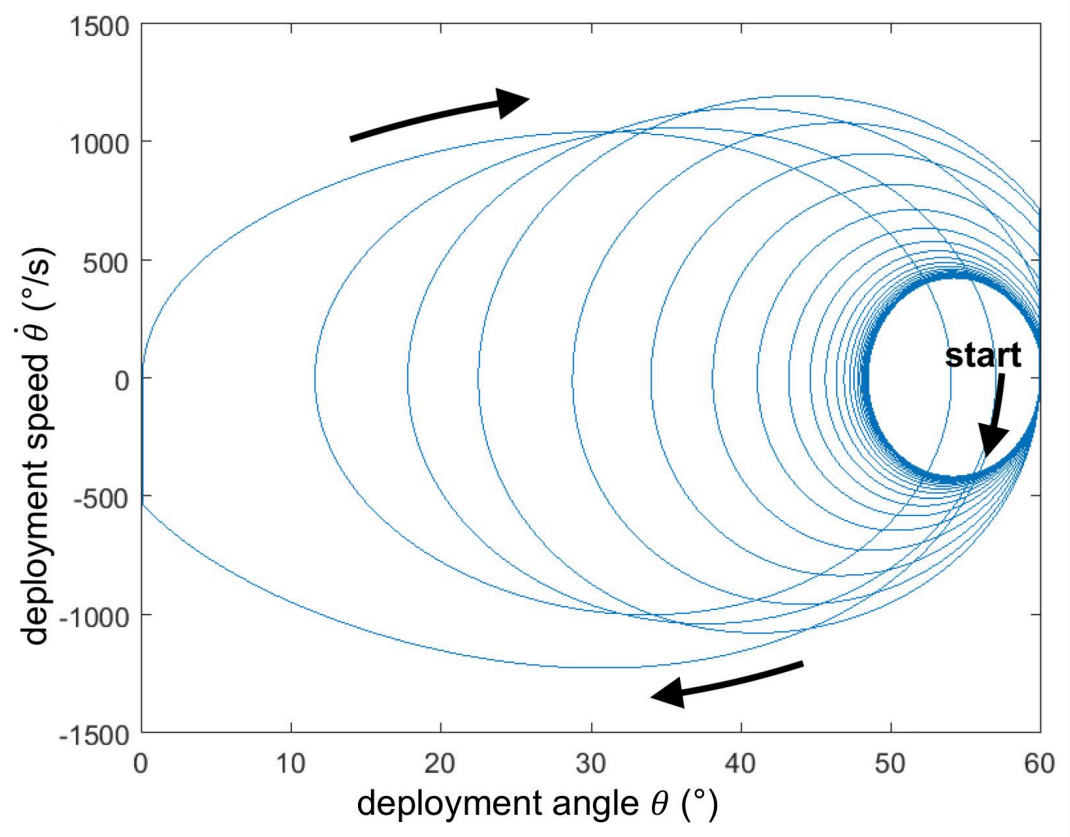

To explain the initial condition and the effect of boundary (at $\theta=0^{\circ}$ and $\theta=60^{\circ}$ ), the text below is added into the paragraph before Eq. 16 and on p8-9: "The initial condition (marked out in Figure 5) assumes a near-fully deployed state with $\theta=0^{\circ}$ and $\dot{\theta}=0^{\circ}$, and $\dot{\theta}$ is set to zero every time when the extreme deployment angle $\left(\theta=0^{\circ}\right.$ or $\left.\theta=60^{\circ}\right)$ is reached, representing an inelastic constraint."

- In Fig. 6 and elsewhere please be consistent with the units. Either use $\wedge\{\mid c i r c\}$ or degrees consistently. Also I would advise the authors to not use the slash symbol [/] to separate the label and the units as it can be confusing.

\section{Reply:}

"/degree" are changed to "(^\{\|circ\})", including Figure 5-11.

- In Fig. 6 how is the normalized torque calculated?

\section{Reply:}

To provide this information, the following underlined text is added to the paragraph before Eq. (16), p8: "The aerodynamic roll 
torque is numerically evaluated using Newtonian method, which assumes the shield to have an ideal origami shape. The result is shown in Figure 6, with its maximum value normalised to unity".

- In Fig. 6 using a contour plot may produce a more legible figure. Currently it is impossible to tell the magnitude $(z)$ of any point $(x, y)$, or where is the line that produces zero torque.

\section{Reply:}

Thanks for your suggestion! Fig. 6 is changed to contour plot, with the zero-torque-line marked out by red line:

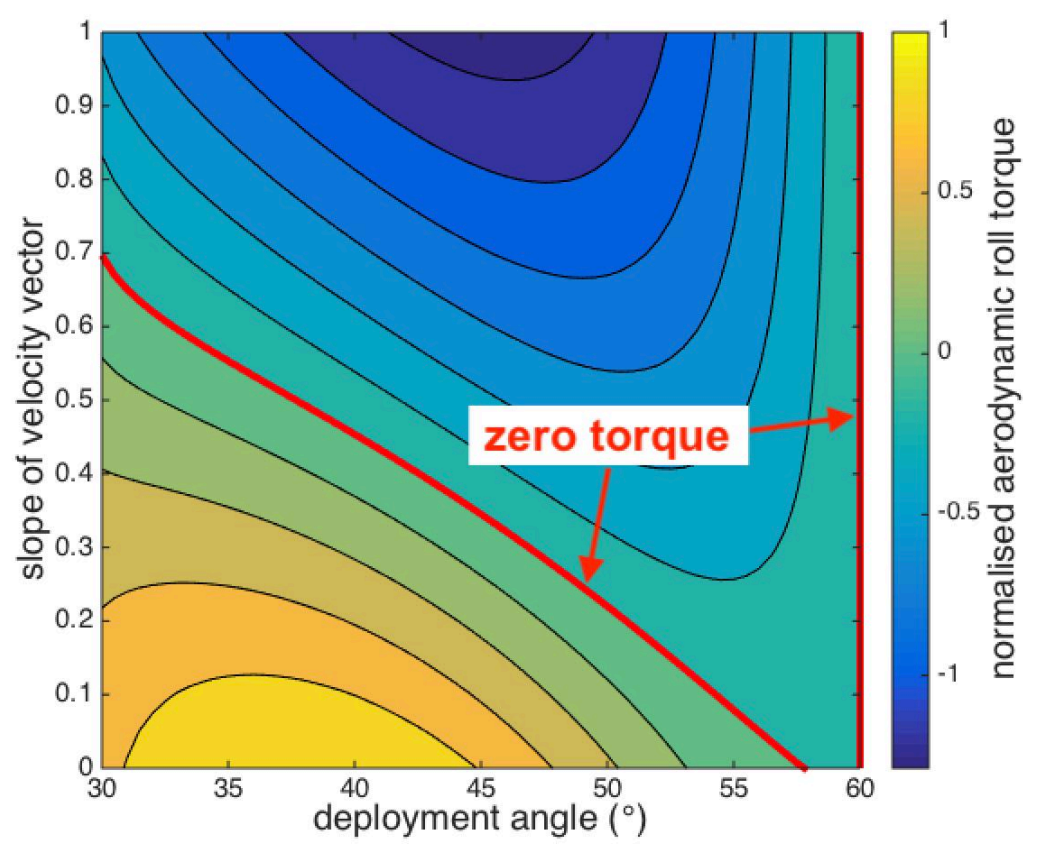

- In a few places in the manuscript write "an unique". My understanding is that the correct form is "a unique".

\section{Reply:}

The manuscript is revised accordingly. Thanks for your help in improving this manuscript! 


\section{Reviewer 2}

Dear Authors,

1. This paper is about a sub-system of a spacecraft to deploy a heat shield for re-entry maneuvers, exploiting rotational motion of the spacecraft caused by the same structure of the shield. I understand that the manuscript is oriented to show a dynamical analysis of the motion during the deployment of the shield. I experienced difficulties to follow and understand the dynamical analysis of your system. Firstly, a more detailed force diagram is required, so the reader could easily understand what are the forces (or torques) you refer to and where they act.

\section{Reply:}

Thanks for your suggestion! The previous force diagram Figure $2 \mathrm{a}$ is revised to show the distributed forces with better clarity, Figure $\mathbf{2 b}$ is added to show the torques as well as the origins of the torques:
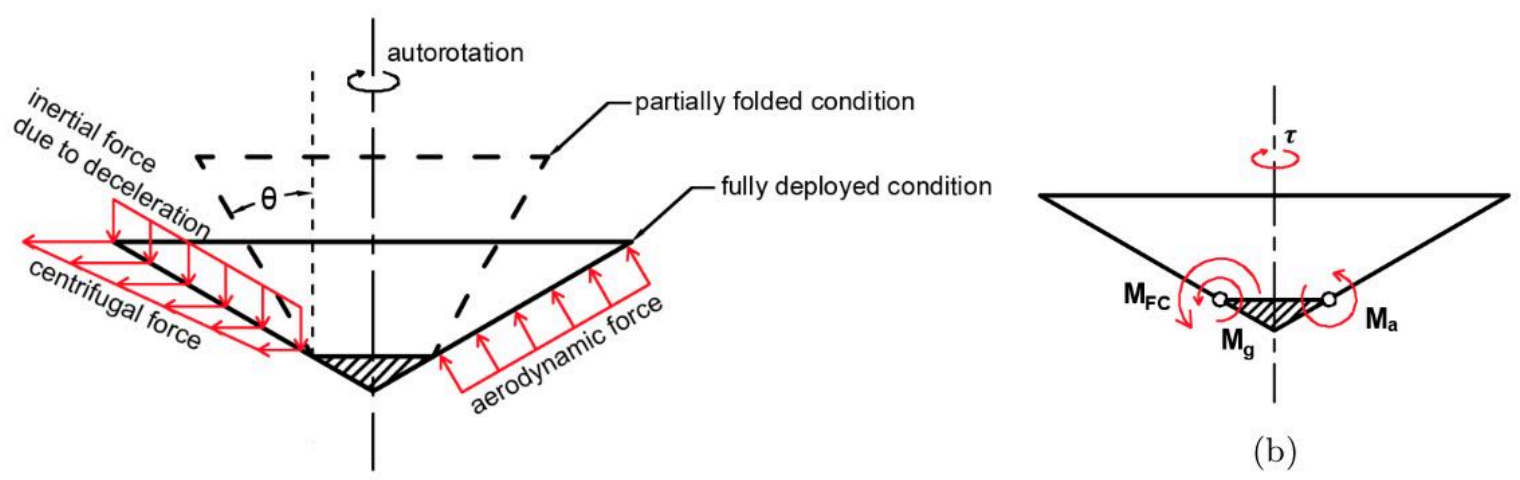

(b)

(a)

Figure 2: Schematic view of the centrifugally deployed heat shield, (a) showing the three types of forces that contribute to the shield's deployment, and (b) the resulting torques with respect to the root of the shield, $M_{F C}, M_{g}$ and $M_{a}$.

2. For a more rigorous analysis, you should also state what coordinate system was considered when writing the equations of torques; and since the torques considered are caused by forces (i.e. not pure couples) you should specify with respect to which point the torques are computed. 


\section{Reply:}

Besides the addition of Figure $\mathbf{2 b}$, to describe the analysis more rigorously, the underlined sentences below are added:

Before Eq. (1), p4: "..., and the anti-deploying moment from aerodynamic load $M_{a}$. The moments are calculated as scalars with respect to the root of the shell and with directions shown in Figure 2b. The total deploying moment $M$ is:",

After Eq. (9), p5: "Where $\tau$ is the roll-torque, and $I_{\text {spin }}$ is the vehicle axial angular inertia. Both are calculated with respect to the vehicle axis, and are functions of $\theta . \tau$ is treated as a scalar, and is set to positive/negative when it accelerates/decelerates the autorotation".

3. All these details are important to end up formulating the equations of motion in a clear manner, showing explicitly the obtained differential equations. I was expecting to see equations that look like $\backslash \operatorname{dot}\{\backslash \operatorname{vec}\{X\}\}=\backslash \operatorname{vec}\{F\} \backslash \operatorname{left}(\backslash \operatorname{vec}\{X\}, \backslash \operatorname{vec}\{u\} \backslash$ right $)$, being $\backslash \operatorname{vec}\{X\}$ your state vector and $\backslash \operatorname{vec}\{u\}$ your control inputs. But in the entire Section 2 "Analysis of the heat shield system" I couldn't find any formulation of the resulting equation of motion for the shield.

Reply:

To include a more complete equation that describes the shell's deploying dynamics, the text below is added after Eq. (7), p5:

"Using the equations above, Equation (2) can be simplified to:

$$
\ddot{\theta} \approx \omega^{2}(0.700 \theta+0.963) \cos \theta-q(9.31 \theta-3.99) \sin \theta
$$

The autorotation of the shield was described by Eq. (9), which is now revised to a more common expression:

$$
\dot{\omega}=\frac{\tau(\theta)}{I_{\text {spin }}(\theta)}
$$


4. You should provide a clearer explanation (with a Force Diagram) what each torque in Eq. (1) is.

\section{Reply:}

A new diagram Figure $2 b$ is added to show the torques:

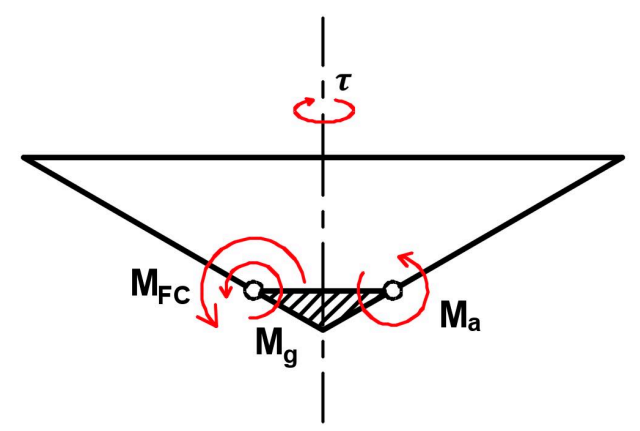

Extra explanation is also added, which is covered by Response 2.

5. Since you build on the heat shield's structural dynamics explained on [20], in order to have this paper more self-contained, you should present a summary of the main statements of that work.

\section{Reply:}

The structural dynamic behaviour explained by [20] was briefly summarised in paragraph 3 of Introduction, p2.

With the revisions made, the paper is now self-contained without requiring further detailed information from reference [20].

6. In the sentence just before Eq. (6), you state that $a_{-}\{d e c\}$ can be derived using Newton's law of motion, thus Mg becomes:

$\mathrm{Mg} \backslash$ propto $\backslash$ rho $\mathrm{v}^{\wedge} 2$ (\theta -0.347$) \sin \backslash$ theta. How this equation is related to Eq. (4) where $\mathrm{Mg}$ is also stated? What is $\mathrm{a}_{-}\{\mathrm{dec}\}$ ? was that expression for $\mathrm{a}_{-}\{\mathrm{dec}\}$ obtained in [20]? Please, make that explicit.

\section{Reply:}

To clarify the statement and eliminate the necessity to mention reference [20] here, this paragraph (on p5) is revised to: 
"Where $\omega$ is the rate of autorotation, $q$ is dynamic pressure, and $a_{d e c}$, the vehicle axial deceleration can be derived using Newton's law of motion:

$$
a_{d e c}=\frac{q S C_{D}}{m} \propto q(\theta-0.347)
$$

Where $S$ is shield reference area, $C_{D}$ is drag coefficient, and $\boldsymbol{m}$ is vehicle mass. Substituting Equation (6) into (4), we have:

$$
M_{g} \propto q(\theta-0.347) \sin \theta \quad \text {, }
$$

7. Right after Eq. (6) it reads: "Since the variation of $\$ \vee \$$ is usually minor...., and is significantly lower than the structural oscillation, the phase lag due to the integration between $\$ a_{-}\{\mathrm{dec}\} \$$ and $\$ \vee \$$ is negligible". I couldn't understand the meaning of this sentence. Firstly, what exactly you are comparing the velocity $\$ \vee \$$ against, when you say that is lower than the structural oscillation? Are you comparing it to the velocity of the oscillation? And what do you mean by "the phase lag due... is negligible" ?

Reply:

I agree that the expression is not clear. This sentence (after Eq. $(8)$, p5) is now trimmed to include only the necessary information: "Since the variation of $v$ is usually minor except in the less-important low-speed regime, the dynamic pressure $q$ can be regarded as constant".

It then contributes to the point that $\theta-M_{g}$ and $\theta-M_{a}$ relationships are near linear.

8. In Eq. (7), I understand that I_\{spin $\}$ is considered constant during the deployment of the shield. Is that the case? Does I_\{spin\} vary during the deployment? 


\section{Reply:}

$I_{\text {spin }}$ varies during the deployment, since it is a function of deployment angle $\theta$. This is clarified by adding the following underlined text after the equation (now is Eq. (9)): "Where $\tau$ is the roll-torque, and $I_{\text {spin }}$ is the vehicle axial angular inertia. Both are calculated with respect to the vehicle axis, and are functions of $\theta^{\prime \prime}$.

\section{Last paragraph of p. 5: "To enable oscillation suppresion, ..." To what} oscillation you refer to? Oscillation of what variables? It is not clear. In the next sentence of the paragraph you mention that the reaction wheel is to stabilize the oscillation of the spin rate. If you refer to oscillations in the spin rate, this should be mentioned before the first sentence, so it's clear to the reader what you are referring to. I was also confused if you were referring to the structural oscillations that you mentioned in the paragraph after Eq (6). Furthermore, if you claim that without the reaction wheels there will be oscillations, it would be very enriching to show them.

\section{Reply:}

\section{Thanks for your suggestion!}

To explain the oscillation earlier in the paper, the $3^{\text {rd }}$ paragraph of Section 3 is moved forward to before the last paragraph of p5:

"Simulations have shown that a limit cycle oscillation of the flexible heat shield exists within nearly the whole flight regime, which leads to variations in $C_{D}$ and thus fluctuates the deceleration. This phenomenon can be seen in Figure 10, where rapid fluctuations in deployment angle (for the uncontrolled scenarios with $a=b=0$ ) are accompanied by fluctuations in deceleration. The vibration may cause damage to a payload especially when it has low natural frequencies (that is close to the oscillation frequency, which is usually $0.1 \mathrm{~Hz} \sim 10 \mathrm{~Hz}$ depending on the flight regime). Thus efforts are made to suppress this oscillation". 
10. In p. 6 , there is an entire paragraph oriented to explain the principles of the shift-phase controller, and within the paragraph the following sentence: "Trails have shown...". I guess that it should read "Trials have shown....". Moreover, how from trials you got that number? what was the criterion used? Please, elaborate on this.

\section{Reply:}

"Trails" is changed to "Trials". Thanks for the correction.

To provide more information on the determination of $K_{T}$, the sentence "Trials have shown ... satisfactory performance" is revised to: "In the present proof-of-concept study, $K_{T}$ is set to 0.25 after a series of trials since it provides effective oscillation suppression with a low requirement on actuator torque".

11. Furthermore, the next sentence: "Such a controller mimics a proportional-differential..., but is less sensitive to noise". Why do you claim that it mimics a PD controller? Is it because of Eq. (8)?. Once again, you should be much clearer when you describe your rational. If it is because of Eq. (8), then first I had to guess that i'_0 denotes the time derivative of $i \_0$. Is it true? You should let the reader know what that notation means. It is not in the nomenclature, nor is a conventionally accepted nomenclature like $\backslash \operatorname{dot}\{\hat{i}\}$. Should we interpret that it mimics a PD controller because the right hand side of Eq. ( 8 ) is "-T0/(2*pi) i'_0 - i_0", which would be a sum of a term including the time derivative of the signal plus other term proportional to the signal? But in these case the constants that multiply the derivative and the proportional terms are not arbitrary, i.e. there is no freedom to pick any value. Maybe your statements are all correct, but the explanation provided is too vague and lacks of rigorousity. Please, elaborate and provide rigorous arguments supported by Math formulation. 


\section{Reply:}

$i^{\prime}$ is changed to the more common expression of $i$.

The description associated with Eq. (11) (which was Eq. (8) before revision) is re-written as below:

"Such a controller mimics a proportional-differential controller when the input is sinusoidal, but is less sensitive to noise. The input spin rate signal has a near sinusoidal component due to the oscillation, and a non-zero component that varies slowly during descent, which represents the autorotation. Therefore, the input spin rate signal can be assumed as $i_{0}=\sin \left(2 \pi t / T_{0}\right)+C(t)$, where $\sin \left(2 \pi t / T_{0}\right)$ is the sinusoidal oscillation, and $C(t)$ is the non-zero component. With $K_{T}=0.25$ the output will be:

$$
\begin{aligned}
i_{1} & =\sin \left(\frac{2 \pi\left(t-0.25 T_{0}\right)}{T_{0}}\right)+C\left(t-0.25 T_{0}\right)-\sin \left(\frac{2 \pi t}{T_{0}}\right)-C(t) \\
& \approx-\cos \left(\frac{2 \pi t}{T_{0}}\right)-\sin \left(\frac{2 \pi t}{T_{0}}\right)=-\frac{T_{0}}{2 \pi} \cdot \dot{i_{0}}-i_{0}
\end{aligned}
$$

Note that the term $C\left(t-0.25 T_{0}\right)-C(t)$ is neglected. According to Equation (11), the output from the phase-shifter is a linear combination of a term containing the time derivative of the oscillation $\left(-T_{0} / 2 \pi \cdot i_{0}\right)$ and another term proportional to the oscillation $\left(-i_{0}\right)$. It thereby functions as a PD controller to suppress this oscillation. $i_{1}$ is then normalised to unity before feeding into the anti-saturation controller.

In fact, the ratio between $P$ ad $D$ gains can be adjusted by tuning $K_{T}$. By re-writing Equation (11) as $i_{1}=-2 \sin \left(K_{T} \pi\right) \cos \left(2 \pi t / T_{0}-K_{T} \pi\right)$, it can be seen that $K_{T} \rightarrow 0$ gives a pure $D$ controller, but is sensitive to noise since $i_{1} \rightarrow 0 . K_{T}=0.5$ gives a pure $P$ controller, which lacks the D component." 
12. In p. 6, the paragraph between Eqs (9) and (10): "The switching gain controllers..." You should introduce that much clearer. It is the first time in the paper that you mention that you are using switching scheduled controllers and that you pursue de-saturation of the reaction wheels. Once again, maybe what you claim is totally correct. But as a reader (and I tried to read it multiple times), I'm having difficulties to understand clearly what you intend to do.

Reply:

To explain the gain-scheduled controllers in a clearer manner, the description of downrange control before Eq. (12), p7 is revised to:

"The downrange controller alters the deployment angle and drag coefficient through gain scheduling, which reduces the throttle when the throttle is negative/positive and thus favours the increment/reduction of vehicle spin rate (Figure 4)."

The description of anti-saturation control, which was located before the downrange control, is now moved to the back, and is revised to:

"Momentum saturation is inevitable when a biased control is applied, which requires an anti-saturation control. As can be seen in Figure 4, the function of the de-saturation gain scheduling is to reduce the throttle when the motor is accelerating (when $i_{1} F>0$ ) and thereby tends to reduce the motor speed"

13. Also in p. 6, it is stated: "The measurement of axial deceleration...., which is explained in details in section 4". In Section 4, it is stated: "The basic mechanism of the reaction wheel controller is already discussed in section 2..." But in Section 2 there are no equations that show the dynamics of the reaction wheel. Once again, without math formulation, the paper lacks of the required rigorousity.

\section{Reply:}

To show the dynamics of the reaction wheel, the following text is added as the $2^{\text {nd }}$ paragraph before Eq. (10) in Section 2, p6: 
"Assume the maximum torque from the reaction wheel has a magnitude of $\tau_{\text {wheel}}$, then the wheel outputs a torque of $i \tau_{w h e e l}$ when the throttle is set to $i(i \in[-1,1])$, and Equation (9) becomes:

$$
\dot{\omega}=\frac{\tau_{\text {aero. }}+i \tau_{\text {wheel }}}{I_{\text {spin }}},
$$

Please note the direction of this torque was defined after Eq. (9).

14. There are also many other minor things like at the end of the first paragraph of Section 4, it reads: "..., which can be approximated by Equation [20]:" At a first glance, it seems that [20] is the number of the equation you are referring to. Since it is a reference, please rephrase the sentence, like saying: "..., which can be approximated by Eq. (14), as stated in [20]". Those very easy changes, make the narrative much easier to follow, for a reader who reads the paper for the first time. This same issue with "[20]" also occurs before Eq. (15). Also, when you refer to an equation, place the number between parenthesis, as is the usual convention.

\section{Reply:}

Thanks for your corrections. The manuscript is revised accordingly, with all the equation numbers placed between parentheses, and citations rearranged where necessary.

15. In summary, respectfully, in my opinion the paper must undergo a major revision to be published. The idea and concepts must be addressed with much more clarity and supported by mathematical or logic statements. It must be proofread very carefully before submission.

\section{Reply:}

Thanks for your help in improving this manuscript! The manuscript has now been revised thoroughly to improve the readability, with the description of the concept further clarified by mathematical statements. 
- Unique manoeuvrability using reaction wheel and centrifugally deployed decelerator

- Limit cycle structural oscillation suppressed using a concise control algorithm

- CubeSat-sized system achieved desired performance during simulated reentry

- Understanding the structural dynamic behaviours of the flexible decelerator 


\title{
Downrange manoeuvre and oscillation suppression of a self-regulating centrifugally deployed flexible heat shield using a controlled reaction wheel
}

\author{
Rui Wu ${ }^{\mathrm{a}, *}$, Peter C.E. Roberts ${ }^{\mathrm{a}}$, Constantinos Soutis ${ }^{\mathrm{b}}$, Carl Diver ${ }^{\mathrm{a}}$ \\ ${ }^{a}$ School of Mechanical, Aerospace and Civil Engineering, The University of Manchester, UK \\ ${ }^{b}$ The University of Manchester Aerospace Research Institute, UK
}

\begin{abstract}
A recent study has introduced a flexible deployable heat shield that passively deploys and stiffens due to centrifugal forces generated from a self-regulated autorotation. This paper demonstrates that the heat shield is similar to a PI controlled second order nonlinear system, which explains why the deployment is accompanied by a limit cycle structural oscillation that persists throughout a simulated re-entry. The heat shield design offers a unique capability to actively adjust the deployment using conventional attitude control devices. This operation is explored by simulating the re-entry of a CubeSat-sized vehicle equipped with an off-the-shelf reaction wheel controlled by a switching phase shift controller and gain-scheduled controllers. The effects of the control parameters are investigated, and successful oscillation suppression as well as an open-loop downrange manoeuvre of over $300 \mathrm{~km}$ is predicted for re-entry from low earth orbit.
\end{abstract}

Keywords:

entry vehicle, deployable aerodynamic decelerator, targeted re-entry, centrifugal deployment, limit cycle oscillation

\section{Nomenclatures}

$a, b$ : downrange control parameters

$a_{d e c}$ : deceleration of vehicle

$C_{D}$ : drag coefficient

$f$ : natural frequency of the oscillatory motion

$F$ : ratio of reaction wheel angular velocity and wheel maximum (saturated) speed

$g$ : gravitational acceleration

$i$ : control signal

I: angular inertia

$l$ : base diameter of the fully deployed heat shield

$m$ : total mass of vehicle

$M$ : total deploying moment

${ }^{*}$ Corresponding author

Email address: rui.wu@manchester.ac.uk (Rui Wu) 
$M_{a}$ : anti-deploying moment from aerodynamic force

$M_{E}$ : anti-deploying moment from elastic force

$M_{F_{C}}$ : deploying moment from centrifugal force

$M_{g}$ : deploying moment from axial deceleration

$N_{\text {torque factor }}$ : Torque factor

$q$ : dynamic pressure

$S$ : reference surface area of the vehicle

St: Strouhal Number

$t$ : time

$T_{0}$ : period of natural oscillation

$v$ : descending velocity

$\theta$ : deployment angle

$\omega$ : spin rate of autorotation

$\rho_{S}$ : shell surface density

$\tau$ : Roll-torque

$C G$ : Centre of Gravity

$I R V E$ : Inflatable Re-entry Vehicle Experiment

\section{Introduction}

Deployable aerodynamic decelerators for atmospheric entry have been attracting increasing attention due to the demand for a next generation Mars entry system for heavy payloads, as well as a small recoverable orbital scientific platform, such as a CubeSat that can de-orbit and recover the payload using a re-entry vehicle [1, 2, 3, 4, 5, 6, 7, 8, 9. The advantages of deployable heat shields over conventional rigid ones mainly come from two aspects [4, 10:

1. Low ballistic ratio (defined as $m / S C_{D}$ ) under the deployed condition leads to a higher deceleration in the upper atmosphere and thus reduced thermal load, as well as providing sufficient deceleration through planetary atmospheres that are less-dense than the Earth's (e.g. Mars), or even soft landing without a parachute system;

2. When stowed, the heat shield can be fitted into a limited space determined by the launch vehicle fairing or the acceptable payload volume.

Current developments mostly focus on two types of structures: the inflatable and the mechanically deployable structures. The inflatable structures are based on flexible thermal protection materials supported by air-tight chambers that deploy and stiffen when inflated by an on-board gas source or ram air [11, 12, 13, 14, 15, 16. The mechanically deployable structures are usually umbrella-like mechanisms consisted of rigid components as the skeleton and flexible thermal protection materials as the skirt [4, 17, 18, 19].

Meanwhile, a new type of centrifugally deployed heat shield has been proposed in a previous study by the authors [20]. The core of the concept is a flexible conical shell made of ceramic fabric. Since local reinforcements are applied along an origami pattern, the shell naturally buckles along the pattern and forms a spiral shape when folded, as shown in Figure 1. The spiral shape generates a roll-torque when descending through an atmosphere and leads to autorotation. In turn, the spin progressively flattens the shell, reducing the induced roll-torque. The rate of autorotation is thereby determined by the shell's deployment condition, and converges to a value where the centrifugal force 
on the flexible shell is in equilibrium with the aerodynamic load, thus enables a self-regulated spin and deployment. In this way, the structure is deployed and stiffened by inertia force generated from motion (i.e. centrifugal force from autorotation) rather than elastic forces from solid or gaseous materials, and as shown by the previous study, it could realise a CubeSat-sized re-entry vehicle that is lightweight, concise and high packing density [20].
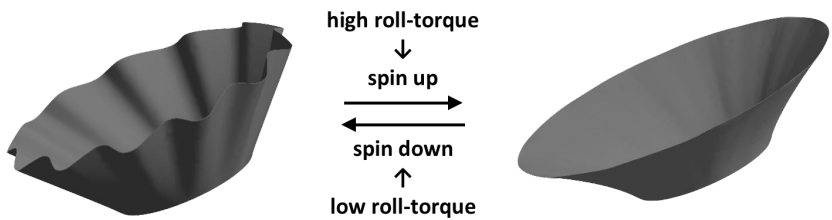

Figure 1: Shape of the centrifugally deployed flexible heat shield generated from FE simulation, showing that equilibrium can be achieved between the partially folded condition (left) and the fully deployed condition (right) when the centrifugal force (due to autorotation) on the shield balances the aerodynamic force [20].

Besides decelerating the payload while surviving aeorthermodynamic heating, advanced missions also require the entry vehicle to be manoeuvrable in order to actively adjust the trajectory and enable a precise landing [21]. For a ballistic vehicle, this is usually achieved using a small lifting force generated by shifting the vehicle Centre of Gravity ( $\mathrm{CG}$ ) or changing the aerodynamic shape. The Inflatable Re-entry Vehicle Experiment (IRVE)-4 of NASA has proposed active CG shift to control the angle of attack of a 3-meter diameter inflatable aeroshell [13. Meanwhile, a number of studies on inflatable aeroshells with the stacked tori configuration similar to the IRVE have demonstrated different ways to either permanently or actively vary the shape of the aeroshell. Some studies have proposed to deform the whole aeroshell by canting the tori 21, sliding the tori perpendicular to the initial axis of symmetry 22,23 , or morphing the tori using actuators 24 . Other concepts includes adding actuated flaps/trim tabs or compressible outer torus to change the aeroshell's centre of pressure [21, 23. On the other hand, mechanically deployable heat shields can also generate lift by having a permanent unsymmetrical shape when deployed [25]. Alternatively, an umbrella-like structure can realise variable frontal area (thus ballistic ratio) by actively controlling the extend of deployment, thus enabling downrange control [17.

In comparison with inflatable and mechanically deployable heat shields, the centrifugally deployed heat shield offers a unique drag modulation capability by actively controlling the extent of deployment using conventional attitude control devices. This is because the centrifugal force, which deploys and stabilises the structure, depends on the vehicle's autorotating (rolling) motion. Therefore, when centrifugal force is altered by actively adjusting the roll rate using an actuator, the heat shield will deploy to a varied extent where it re-balances with the aerodynamic load, and effectively changes deployed drag area and therefore the trajectory.

Nevertheless, the reliance on centrifugal force and the structural flexibility also leads to problems. Lifting manoeuvres are not feasible since a non-zero angle of attack can not be maintained without conning motion as a result of gyroscopic effects on the rotating vehicle. A previous numerical study has also revealed an oscillatory deploying-folding motion of the undamped heat shield throughout the descent 20 .

The present work is a proof-of-concept study that focuses on utilising a reaction wheel to actively manipulate the extend of deployment and therefore, provides a way to suppress the oscillation. The feasibility to adjust the downrange trajectory using such a system is also discussed. 

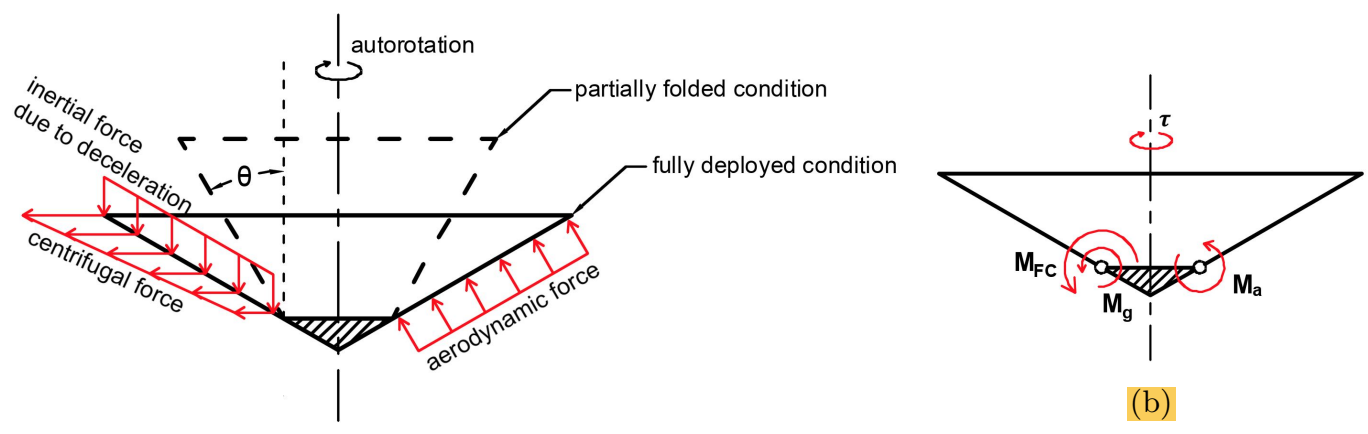

(b)

(a)

Figure 2: Schematic view of the centrifugally deployed heat shield, (a) showing the three types of forces that contribute to the shield's deployment, and (b) the resulting torques with respect to the root of the shield, $M_{F C}, M_{g}$ and $M_{a}$.

\section{Analyses on the heat shield system}

The proposed control method uses a single reaction wheel to adjust the rate of autorotation and thereby influence the deployment angle. Therefore, the analyses focus on the deploying-folding motion of the flexible heat shield as well as the autorotation of the vehicle, and all the analyses reported here are based on an aeroshell pointing stably into the flow with zero angle of attack, while the pitching and yawing motions as well as the gyroscopic effects from the autorotation are not considered. For a preliminary study, this is a reasonable simplification since the objective is to reveal the baseline effect of the proposed control, whilst the six Degree-of-Freedom (DoF) flight dynamics requires significantly more computational resource and is not analysed here.

During entry, the deployment of the heat shield is determined by three moments of forces (Figure 2): the deploying moment due to centrifugal force $M_{F_{C}}$, the deploying moment due to axial deceleration $M_{g}$, and the anti-deploying moment from aerodynamic load $M_{a}$. The moments are calculated as scalars with respect to the root of the shell and with directions shown in Figure 2b. The total deploying moment $M$ is:

$$
M=M_{F_{C}}+M_{g}-M_{a}
$$

Then the shell deployment angle $\theta$ can be expressed as below, where $I_{\text {deploy }}$ is shell's inertia associated with the deploying/folding motion. The shell is therefore a second order dynamic system.

$$
\theta=\iint \frac{M}{I_{\text {deploy }}} \cdot d t \cdot d t
$$

Based on the understanding of the heat shield's structural dynamics [20], a block diagram of the heat shield is constructed, as shown in Figure 3. Ignoring the active control from the reaction wheel controller, the heat shield can be treated as a second order system regulated by feedback from the three deploying/anti-deploying moments $M_{F_{C}}, M_{g}$, and $M_{a}$ [20]: 


$$
\begin{aligned}
M_{F_{C}} & \propto \omega^{2}(\theta+1.38) \cos \theta \\
M_{g} & \propto a_{d e c} \sin \theta \\
M_{a} & \propto q(\theta-0.407) \sin \theta
\end{aligned}
$$

Where $\omega$ is the rate of autorotation, $q$ is dynamic pressure, and $a_{d e c}$, the vehicle axial deceleration can be derived using Newton's law of motion:

$$
a_{\text {dec }}=\frac{q S C_{D}}{m} \propto q(\theta-0.347)
$$

Where $S$ is shield reference area, $C_{D}$ is drag coefficient, and $m$ is vehicle mass. Substituting Equation (6) into (4), we have:

$$
M_{g} \propto q(\theta-0.347) \sin \theta
$$

Using the equations above, Equation (2) can be simplified to:

$$
\ddot{\theta} \approx \omega^{2}(0.700 \theta+0.963) \cos \theta-q(9.31 \theta-3.99) \sin \theta
$$

Since the variation of $v$ is usually minor except in the less-important low-speed regime, the dynamic pressure $q$ can be regarded as constant. Meanwhile, since $\theta$ usually has values between $\pi / 6$ and $\pi / 3\left(30^{\circ} \sim 60^{\circ}\right)$, the $\theta-M_{g}$ and $\theta-M_{a}$ relationships are near linear (Equations (4) and (5)), $M_{g}$ and $M_{a}$ can be treated as Proportional (P) control. $M_{F_{C}}$ contains an Integral (I) control component since $\omega$ is a result of integrated aerodynamic torque:

$$
\dot{\omega}=\frac{\tau(\theta)}{I_{\text {spin }}(\theta)}
$$

Where $\tau$ is the roll-torque, and $I_{\text {spin }}$ is the vehicle axial angular inertia. Both are calculated with respect to the vehicle axis, and are functions of $\theta . \tau$ is treated as a scalar, and is set to positive/negative when it accelerates/decelerates the autorotation.

Therefore, the whole system is similar to a second order system regulated by a PI controller, while analytical analysis is difficult due to the complexity and non-linearity. According to Figure 3 , the behaviour of the system can be influenced by three parameters: the vehicle mass, the origami pattern, and the atmospheric density.

Simulations have shown that a limit cycle oscillation of the flexible heat shield exists within nearly the whole flight regime, which leads to variations in $C_{D}$ and thus fluctuates the deceleration. This phenomenon can be seen in Figure 10, where rapid fluctuations in deployment angle (for the uncontrolled scenarios with $a=b=0$ ) are accompanied by fluctuations in deceleration. The vibration may cause damage to a payload especially when it has low natural frequencies (that is close to the oscillation frequency, which is usually $0.1 \mathrm{~Hz} \sim 10 \mathrm{~Hz}$ depending on the flight regime). Thus efforts are made to suppress this oscillation.

To enable oscillation suppression, spin rate and axial deceleration are measured and fed into the reaction wheel controller, which controls a reaction wheel that exerts an axial torque to stabilise the oscillation of spin rate and thus stabilise the deployment angle. Spin rate measurement is used to indicate the deployment angle, since the latter is difficult to measure, and the oscillation of the two are strongly coupled (due to the dependence of spin angular inertia on deployment angle, Equation 


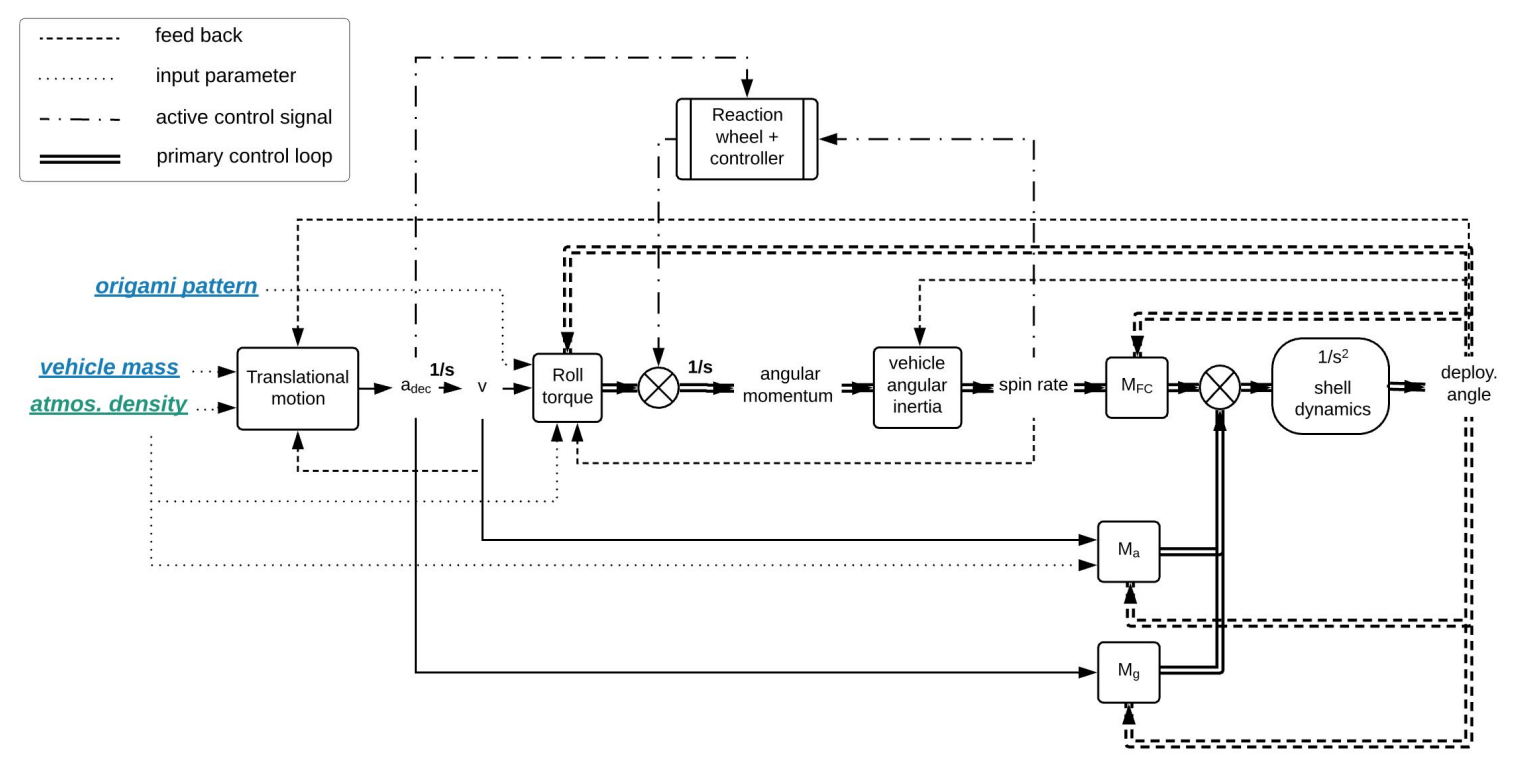

Figure 3: Block diagram of the heat shield assuming zero angle of attack, with the primary feedback loops emphasised by double lines.

(9)) with nearly no phase difference (since the dynamics of the shell deployment is significantly faster than the vehicle spinning dynamics, or in other words, the integral gain is low). The axial deceleration is used to compute a control parameter as discussed later.

Similarly, the reaction wheel is also used to adjust the overall deployment angle and enable open-loop downrange manoeuvre through drag modulation. This is achieved by varying the overall angular momentum of the system to increase/decrease $M_{F_{C}}$, which leads to a biased deployment condition. Aerodynamic drag is thereby manipulated as it depends on the deployment angle. To be noted, the present work only demonstrates the feasibility to achieve extended/shortened downrange trajectory, while the control method to carry out a guided re-entry is not discussed.

Assume the maximum torque from the reaction wheel has a magnitude of $\tau_{w h e e l}$, then the wheel outputs a torque of $i \tau_{\text {wheel }}$ when the throttle is set to $i(i \in[-1,1])$, and Equation (9) becomes:

$$
\dot{\omega}=\frac{\tau_{\text {aero. }}+i \tau_{\text {wheel }}}{I_{\text {spin }}}
$$

The detailed control strategy is shown in Figure 4. Phase shift control is an existing method that has been used to suppress the thermal-acoustic oscillation of gas turbines. Its popularity comes from its simplicity, and thus the ability to survive in harsh environment, as well as the low requirement for knowledge of the the complicated and highly non-linear system [26, 27. For the same reason it is proposed for oscillation suppression of the present heat shield, as it is a nonlinear system that requires the control power and complexity to be minimised. The phase shift controller simply applies a time delay $K_{T} \cdot T_{0}$ to the input spin rate signal, and then outputs the difference between the original and the delayed signal (Figure 4). The measurement of axial deceleration is used to determine the control parameter $T_{0}$ for the phase shift controller, which is explained in details in section 4. In the present proof-of-concept study, $K_{T}$ is set to 0.25 after a 
series of trials since it provides effective oscillation suppression with a low requirement on actuator torque. Such a controller mimics a proportional-differential controller when the input is sinusoidal, but is less sensitive to noise. The input spin rate signal has a near sinusoidal component due to the oscillation, and a non-zero component that varies slowly during descent, which represents the autorotation. Therefore, the input spin rate signal can be assumed as $i_{0}=\sin \left(2 \pi t / T_{0}\right)+C(t)$, where $\sin \left(2 \pi t / T_{0}\right)$ is the sinusoidal oscillation, and $C(t)$ is the non-zero component. With $K_{T}=0.25$ the output will be:

$$
\begin{aligned}
i_{1} & =\sin \left(\frac{2 \pi\left(t-0.25 T_{0}\right)}{T_{0}}\right)+C\left(t-0.25 T_{0}\right)-\sin \left(\frac{2 \pi t}{T_{0}}\right)-C(t) \\
& \approx-\cos \left(\frac{2 \pi t}{T_{0}}\right)-\sin \left(\frac{2 \pi t}{T_{0}}\right)=-\frac{T_{0}}{2 \pi} \cdot \dot{i_{0}}-i_{0}
\end{aligned}
$$

Note that the term $C\left(t-0.25 T_{0}\right)-C(t)$ is neglected. According to Equation (11), it can be seen that the output from the phase-shifter is a linear combination of a term containing the time derivative of the oscillation $\left(-\frac{T_{0}}{2 \pi} \cdot \dot{i_{0}}\right)$ and another term proportional to the oscillation $\left(-i_{0}\right)$. It thereby functions as a PD controller to suppress this oscillation. $i_{1}$ is then normalised to unity before feeding into the anti-saturation controller.

In fact, the ratio between $\mathrm{P}$ ad $\mathrm{D}$ gains can be adjusted by tuning $K_{T}$. By re-writing Equation (11) as $i_{1} \approx-2 \sin \left(K_{T} \pi\right) \cos \left(2 \pi t / T_{0}-K_{T} \pi\right)$, it can be seen that $K_{T} \rightarrow 0$ gives a pure $\mathrm{D}$ controller, but is sensitive to noise since $i_{1} \rightarrow 0 . K_{T}$ gives a pure $\mathrm{P}$ controller, which lacks the $\mathrm{D}$ component.

A major drawback of phase shift controllers is that they may induce the so called secondary peak, or in other words, the instability induced by the controller in frequencies away from the operation frequency [28, 29. In the present research, such instability is observed, but is a nonessential problem considering its low amplitude, as further discussed in section 4.

The downrange controller alters the deployment angle and drag coefficient through gain scheduling, which reduces the throttle when the throttle is negative/positive and thus favours the increment/reduction of vehicle spin rate (Figure 4):

$$
\begin{array}{ll}
i_{3}=a i_{2} & \text { if } \quad i_{2}>0 \\
i_{3}=b i_{2} & \text { if } \quad i_{2} \leq 0
\end{array}
$$

Downrange is shortened when $a>b$, which favours the increment of spin rate and thereby facilitates deployment and leads to a higher aerodynamic drag.

Momentum saturation is inevitable when a biased control is applied, which requires an antisaturation control. As can be seen in Figure 4 the function of the de-saturation gain scheduling is to reduce the throttle when the motor is accelerating (when $i_{1} F>0$ ) and thereby tends to reduce the motor speed:

$$
\begin{array}{ll}
i_{2}=i_{1}(1-|F|) & \text { if } i_{1} F>0 \\
i_{2}=i_{1} & \text { if } i_{1} F \leq 0
\end{array}
$$

Where $i_{1}$ is the output from the phase shift controller, and $F$ is the ratio of the wheel angular velocity and wheel maximum (saturated) speed, as shown in Figure 4 In this way, the controller tends to bring the wheel speed to zero and thereby prevents momentum saturation.

The simulated effects of the controller are discussed with more details in section 4 and 5. 


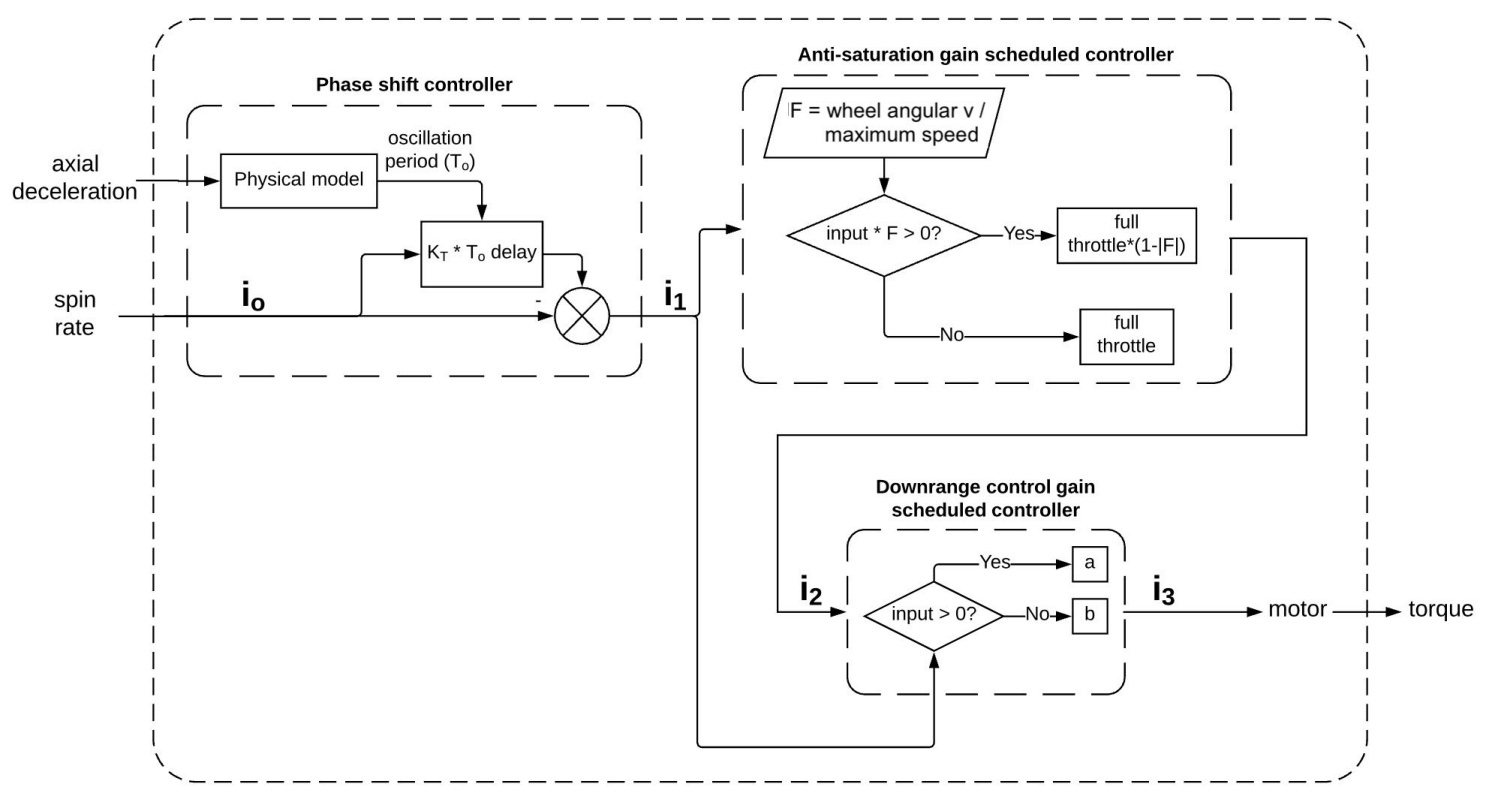

Figure 4: Control algorithm of the reaction wheel controller.

\section{Parameter study on the oscillatory behaviour}

The results discussed in the present research are generated from a numerical simulator developed in Simulink. The simulator is based on Newtonian aerodynamic equations while assuming zero angle of attack, and Newton's second law is used to describe the point mass dynamics of the vehicle. The previous structural dynamic model is also incorporated to include the deploying/folding behaviours of the flexible shield [20]. It should be noted that the Newtonian method only provides a rough estimation of the base line behaviour for subsonic flight conditions. All the results are generated with a maximum time step of $0.5 \mathrm{~ms}$ using a variable-step continuous explicit solver (ode45).

It also worth noticing that the simulator has not included structural damping since damping depends on various unknown factors such as specific material selection and layup. This could lead to an overestimation of oscillation amplitude. However, the structural damping in the design is expected to be weak considering the low heat shield thickness $(\sim 1 \mathrm{~mm})$.

A typical limit cycle oscillation observed in the simulations is shown in Figure 5 , which is the result on a CubeSat-sized vehicle with the baseline design [20, $3 \mathrm{~kg}$ entry mass, and under a flight condition at $30 \mathrm{~km}$ altitude during re-entry from LEO. The initial condition (marked out in Figure 5) assumes a near-fully deployed state with $\theta=0^{\circ}$ and $\dot{\theta}=0^{\circ}$, and $\dot{\theta}$ is set to zero every time when the extreme deployment angle $\left(\theta=0^{\circ}\right.$ or $\left.\theta=60^{\circ}\right)$ is reached, representing an inelastic constraint. Referring to Figure 3 running cases which neglect $M_{g}$ and the coupling between deployment angle and spin angular inertia (by setting $I_{\text {spin }}$ to constant, according to Equation (9p) shows that the oscillation is unrelated to these factors. In fact, the oscillatory behaviour is due to the integration component that determines $M_{F_{C}}$ (equation (99). The aerodynamic roll torque is numerically evaluated using Newtonian method, which assumes the shield to have an ideal origami shape. The result is shown in Figure 6, with its maximum value normalised to unity. The torque 


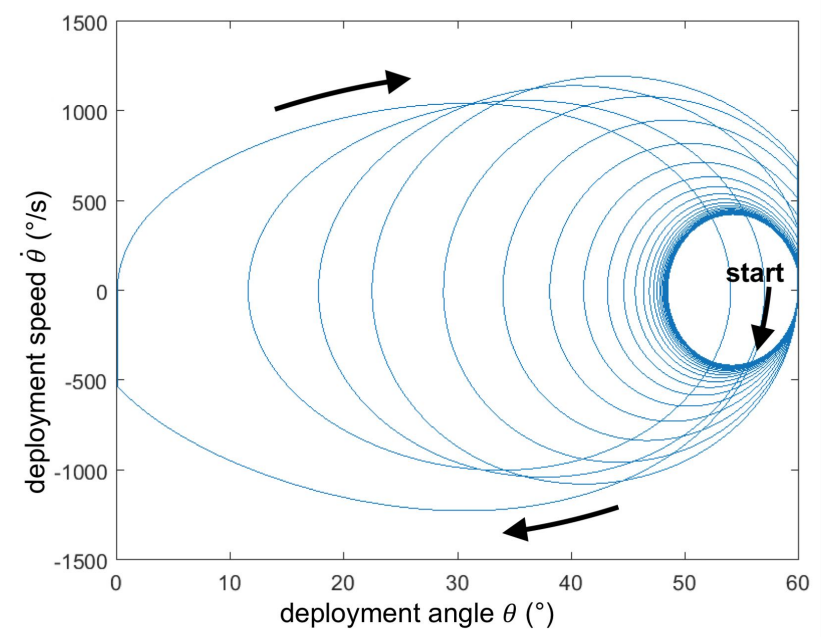

Figure 5: A typical limit cycle oscillation from the simulations, where the deployment speed is the derivative of the deployment angle.

depends on deployment angle and the slope of flow velocity vector with reference to the rim of the heat shield. It worth noticing that this slope is proportional to the dimensionless Strouhal Number:

$$
S t=\frac{\omega \cdot l}{v}
$$

It can be seen from Figure 6 that the aerodynamic roll-torque is generally inversely-related to deployment angle. Therefore, when deployment angle is instantaneously increased from an equilibrium condition (where $\tau=0$ thus $\dot{\omega}=0$ under static condition) due to oscillation, the roll-torque $\tau$ becomes negative. This negative torque then integrates over half oscillation period to reduce spin rate (equation (9p) and thus deployment angle with a phase lag, which assists the oscillation. Meanwhile, the variation of velocity vector slope is minor during the oscillation and thus has minor effect. Therefore, the primary feedback loop in Figure 3 that contains $M_{F_{C}}$, or in other words, the integral component is the cause of the oscillation.

The three factors that determine the actual behaviour of the heat shield, as illustrated in Figure 3, are listed below. Their effects on the oscillation are studied using simulations.

(1) Atmospheric density; simulation assumes Earth atmosphere with density values ranging from $150 \mathrm{~km}$ altitude to sea level;

(2) Characteristics of the origami pattern; in the simulation, the magnitude of aerodynamic roll torque varies from 0.25 to 2 times of the baseline design;

(3) Total mass of the vehicle; varies from $0.75 \mathrm{~kg}$ to $3 \mathrm{~kg}$.

At each data point, the simulation assumes constant atmospheric density and runs until the magnitude and amplitude of deployment angle are stabilised. The simulator also assumes the vehicle axis to be along gravity unlike the real trajectory conditions which have shallow flight path angles at high altitudes. This simplification is made since the objective of this study is to reveal the basic dynamic behaviour of the heat shield, rather than the exact behaviour at various trajectory conditions. 


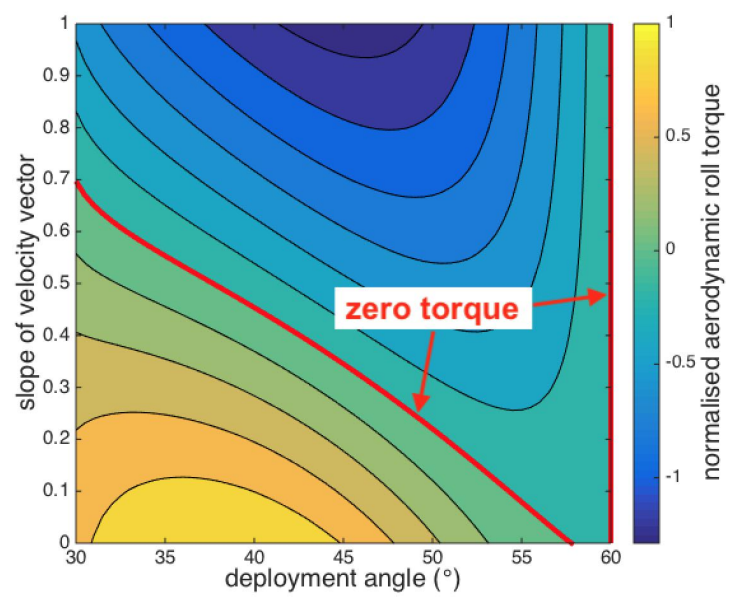

Figure 6: Evaluation of the axial aerodynamic roll torque (with normalised magnitude) using deployment angle and the slope of velocity vector (i.e. the Strouhal number).

The effect of factors (1), (2) and (3) are shown in Figure 7, where torque factor is a value that is multiplied to the baseline aerodynamic roll torque to manipulate its magnitude: $\tau_{\text {simulated }}=$ $N_{\text {torque factor }} \cdot \tau$. It can be seen that the limit cycle oscillation exists throughout the flight regime, while the amplitude usually increases during descent. Generally, increasing torque factor leads to higher oscillation amplitude. This is because a higher torque factor is equivalent to a higher gain in the integral component that causes the oscillation (equation (9)).

The figure also show that the oscillation amplitude is usually higher in denser atmosphere. This is simply due to the fact that the simulator assumes the speed of the deploying motion becomes zero every time it reaches maximum/minimum deployment angle $\left(60^{\circ} / 30^{\circ}\right)$, thus the oscillation amplitude is mechanically constrained. Therefore, the amplitude can be higher when the equilibrium deployment angle is further away from $30^{\circ}$ and $60^{\circ}$, which is the case in dense atmosphere. Similarly, in dense atmosphere there is a trend of increasing oscillation amplitude with decreasing vehicle mass, which is particularly notable in Figure 7b. This is because a lower mass leads to a higher equilibrium deployment angle that is further away from $30^{\circ}$ and thus leads to higher oscillation amplitude.

Figure 7 has also shown another trend of reducing oscillation amplitude due to decreasing vehicle mass, which exists at all the altitudes. This is because a lower mass leads to lower dynamic pressure, which, similar to a reducing torque factor, is equivalent to reducing the integration gain that causes the oscillation.

\section{Oscillation suppression}

The basic mechanism of the reaction wheel controller is already discussed in section 2 the controller feeds back and regulates the spin rate to alter the deployment angle. The control parameter $T_{0}$ in the phase shift controller is the period of the heat shield's natural oscillation, which can be 

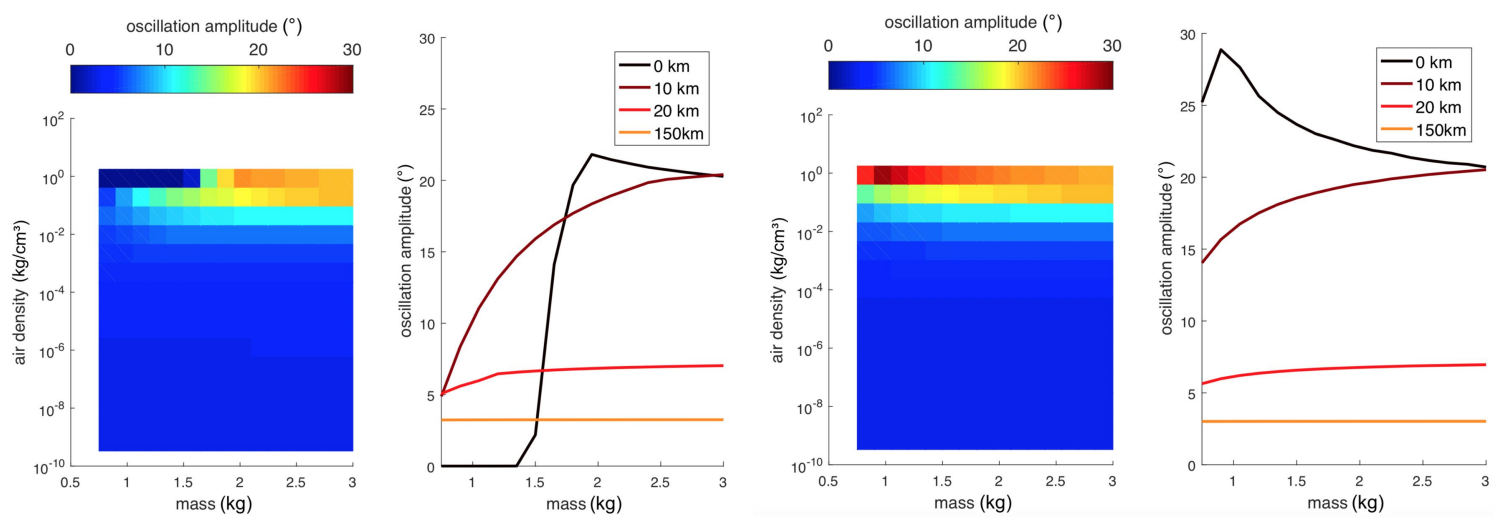

(a) Torque factor $=0.5$

(b) Torque factor $=2$

Figure 7: Effect of vehicle mass, atmospheric density (or equivalent altitude on Earth) and magnitude of aerodynamic torque on the oscillation amplitude of deployment angle; it can be seen that the oscillation is common throughout different flight conditions, while the effects that reduces aerodynamic roll-torque always lead to a lower oscillation except at very low altitude $(<10 \mathrm{~km})$.

approximated by Equation (17), as stated in [20:

$$
f=\frac{1}{2 \pi} \sqrt{\frac{\frac{\partial M}{\partial \theta}}{I_{\text {deploy }}}}
$$

Where $f$ is the natural frequency, $M$ is the total deploying moment on the flexible shell evaluated according to equation (1), $\theta$ is deployment angle, and $I_{\text {deploy }}$ is the deploying angular inertia of the shell. After simplification and assuming a deployment angle close to $60^{\circ}$, equation (17) becomes [20]:

$$
f \approx 0.49 \sqrt{\frac{a_{d e c}\left(1.53 m-1.21 l^{2} \rho_{S}\right)}{l^{3} \rho_{S}}}
$$

Where $a_{d e c}$ is deceleration (including the component of gravity along the vehicle's axis, in $\mathrm{m} / \mathrm{s}^{2}$ ), $l$ is the heat shield base diameter when deployed, $m$ is the vehicle's mass $(\mathrm{kg})$, and $\rho_{S}$ is the surface density of the shell $\left(\mathrm{kg} / \mathrm{m}^{2}\right)$. The vehicle analysed in this section has a total mass of $3 \mathrm{~kg}$, a surface density of $1.1 \mathrm{~kg} / \mathrm{m}^{2}$ and a base diameter of $0.7 \mathrm{~m}$. Thus, equation (17) becomes:

$$
f \approx 1.60 \sqrt{a_{d e c}}
$$

equation 19 provides simple predictions with the error $<25 \%$ in comparison with simulation, and is incorporated into the reaction wheel controller.

Meanwhile, it should be noted that equation (11) ignores the elasticity and damping of the flexible shell, which is difficult to predict as it depends on the actual material and structural properties, whilst the elastic forces also depend on the initial deployment angle at the unloaded condition. However, the effect of elastic and damping force is expected to be weak as the aim of this heat shield design is to eliminate the dependence on elasticity while taking advantage of the structure 
supported by inertia force. Nevertheless, the anti-deployment moment of elastic force, $M_{E}$, is included in the numerical simulators since it has notable effect on the heat shield's deployment angle at high altitude where the aerodynamic effect is weak. Thus equation (1) becomes:

$$
M=M_{F_{C}}+M_{g}-M_{a}-M_{E}
$$

Where $M_{E}$ is evaluated based on the bending rigidity of the shield's root using linear beam theory by assuming a shell thickness of $1 \mathrm{~mm}$, a Young's modulus of $5 \mathrm{MPa}$, and assumes bending occurs in a region within $10 \mathrm{~mm}$ from the shell's root. This provides a reasonable example to show the effect of elasticity, but is not based on any real designs. The deployment angle of the heat shield at unloaded condition is set to the minimum deployment angle $\left(30^{\circ}\right)$, thus the elasticity provides an anti-deployment effect. The effect of $M_{E}$ is illustrated by the trajectory simulation in Figure 10. where the result on an uncontrolled vehicle without accounting for $M_{E}$ is set in comparison with the same vehicle with $M_{E}$. It can be seen that the elastic force has notably reduced the deployment angle at high altitude and therefore increased the downrange, while its effect on the oscillatory behaviour is insignificant. Meanwhile, the spin rate at high altitude is increased by adding $M_{E}$ since the heat shield requires higher centrifugal force to maintain its equilibrium deployment angle.

The sensors in the reaction wheel controller are set to have a sample rate of $1000 \mathrm{~Hz}$, which provides a near ideal scenario. The controller output at $1000 \mathrm{~Hz}$ is then transferred to $50 \mathrm{~Hz}$ by averaging in every $20 \mathrm{~ms}$, thus the motor is controlled at a rate of $50 \mathrm{~Hz}$, and with its torque response assumed to be instantaneous for simplicity. As spin rate varies due to oscillation, the evaluated parameter $T_{0}$ is smoothed by a simple moving average filter with the length of 1000 to prevent the interference caused by this oscillation. This filter has shown no adverse effect since $T_{0}$ changes slowly through descent and is not sensitive to phase lag. With this filter, the noise in deceleration is generally irrelevant to the controller's performance and is not considered as an issue. However, the spin rate measurement could have an excessive noise spectral density in the magnitude of $0.01^{\circ} / \mathrm{s} / \sqrt{\mathrm{Hz}}$ (according to the gyro of a commercial IMU). This could be resolved using redundant sensors and signal processing techniques, but is not considered as the subject of this paper. Therefore, no noise is included in the simulations.

Since the simulation uses a baseline CubeSat-sized vehicle design, a reaction wheel is chosen accordingly to fit within such a system: the Sinclair Interplanetary RW3-0.060, which outputs torque of approx. $\pm 20 \mathrm{mNm}$ with the wheel momentum within $\pm 0.18 \mathrm{Nms}$. Peak performance is achieved at roughly $25 \mathrm{~W}$ and $28 \mathrm{~V}$. The reaction wheel assembly has a total mass of $226 \mathrm{~g}$ and a size of $77 \mathrm{~mm} \times 65 \mathrm{~mm} \times 38 \mathrm{~mm} 30$.

To investigate the basic behaviours of the controlled vehicle, the simulator used to construct Figure 7 is revised to include $M_{E}$, the reaction wheel controller, and the flight path angles that are determined using full trajectory simulation (Figure 10). In order to eliminate the effect of initial conditions, the reaction wheel controller is turned on from the simulation time of $150 \mathrm{~s}$ when the spin rate is generally stabilised. The results at different altitudes are shown in Figure 8, It can be seen that at lower altitude the oscillations are rapidly suppressed after the controller is enabled, whereas at higher altitudes where the oscillation suppression is slower since the phase shift controller becomes ineffective due to the large error in control parameter $T_{0}$. This is because equation $(19)$ no longer holds when the dynamic pressure is low and elasticity has considerable effect on the shell's natural frequency.

Meanwhile, a small residual oscillation always emerge after the suppression of the original oscillation, which is caused by the coupling between the phase shift controller and the heat shield system, and has a frequency higher than the structural natural oscillation. The details of a typical 

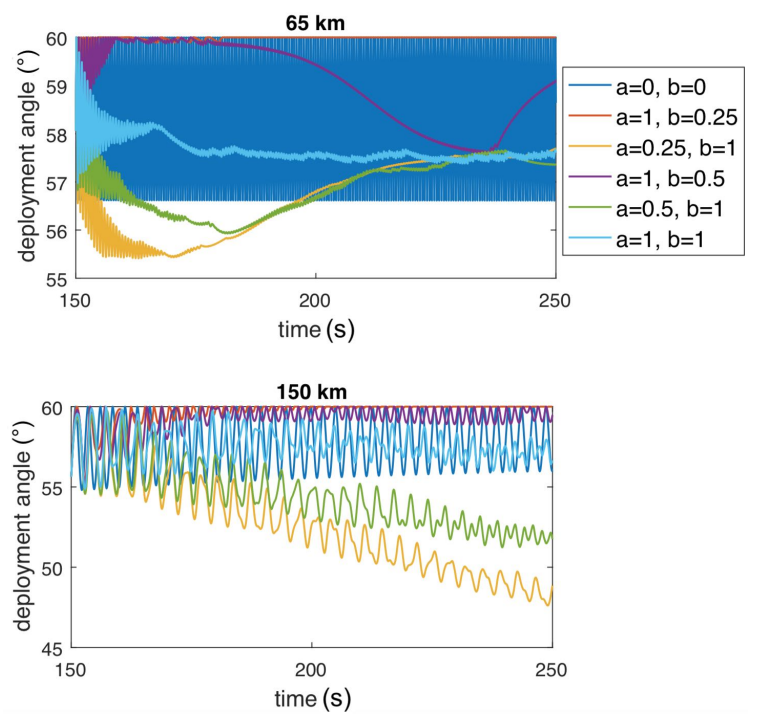

Figure 8: Effect of the reaction wheel controller with various downrange control gains $(a$ and $b)$ at different altitudes, the controller is enabled at the simulation time of $150 \mathrm{~s}$ when equilibrium is reached, it can be seen that oscillation suppression is effective at lower altitude (in comparison with the dark blue line, which is the uncontrolled scenario with $a=0, b=0$ according to Equation (12)-(13)), while downrange control (with $a \neq b$ ) has effectively varied the average deployment angle before momentum saturation.

residual oscillation is shown in Figure 9 This phenomena is reminiscent of the so called secondary peaks caused by phase shift controllers used to suppress thermal acoustic oscillations of gas turbines [28. However, this is not a critical issue considering the low oscillation amplitude, especially during the flight regime with higher dynamic pressure.

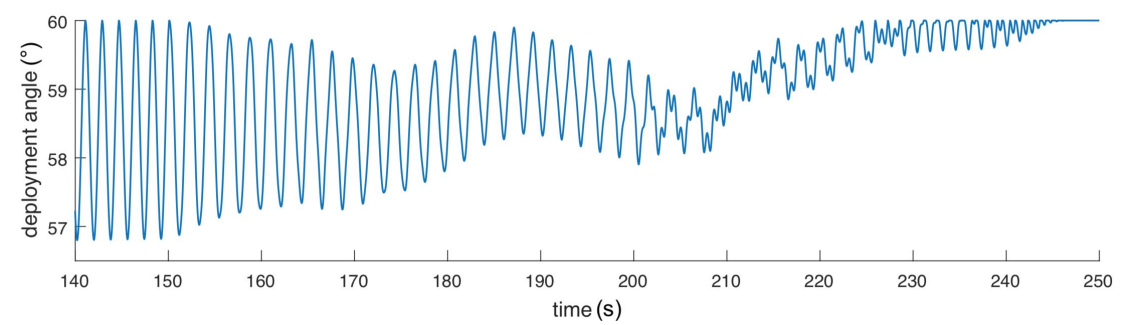

Figure 9: Details of a typical residual oscillation, showing secondary peaks at a frequency higher than the natural oscillation.

Then, the effect of oscillation suppression on the re-entry trajectory is assessed using the trajectory simulator, which assumes a re-entry starting from $15 \mathrm{~km}$ altitude at a velocity of $7800 \mathrm{~m} / \mathrm{s}$, with zero flight path angle, zero spin rate, and an initial deployment angle of $30^{\circ}$. Simulations are terminated when the vehicle reaches $30 \mathrm{~km}$ altitude since the vehicle has already entered the uncritical low speed regime at this altitude.

According to the results shown in Figure 10, the controller leads to effective oscillation reduction in both deployment angle and deceleration throughout descent. From the history of reaction wheel 

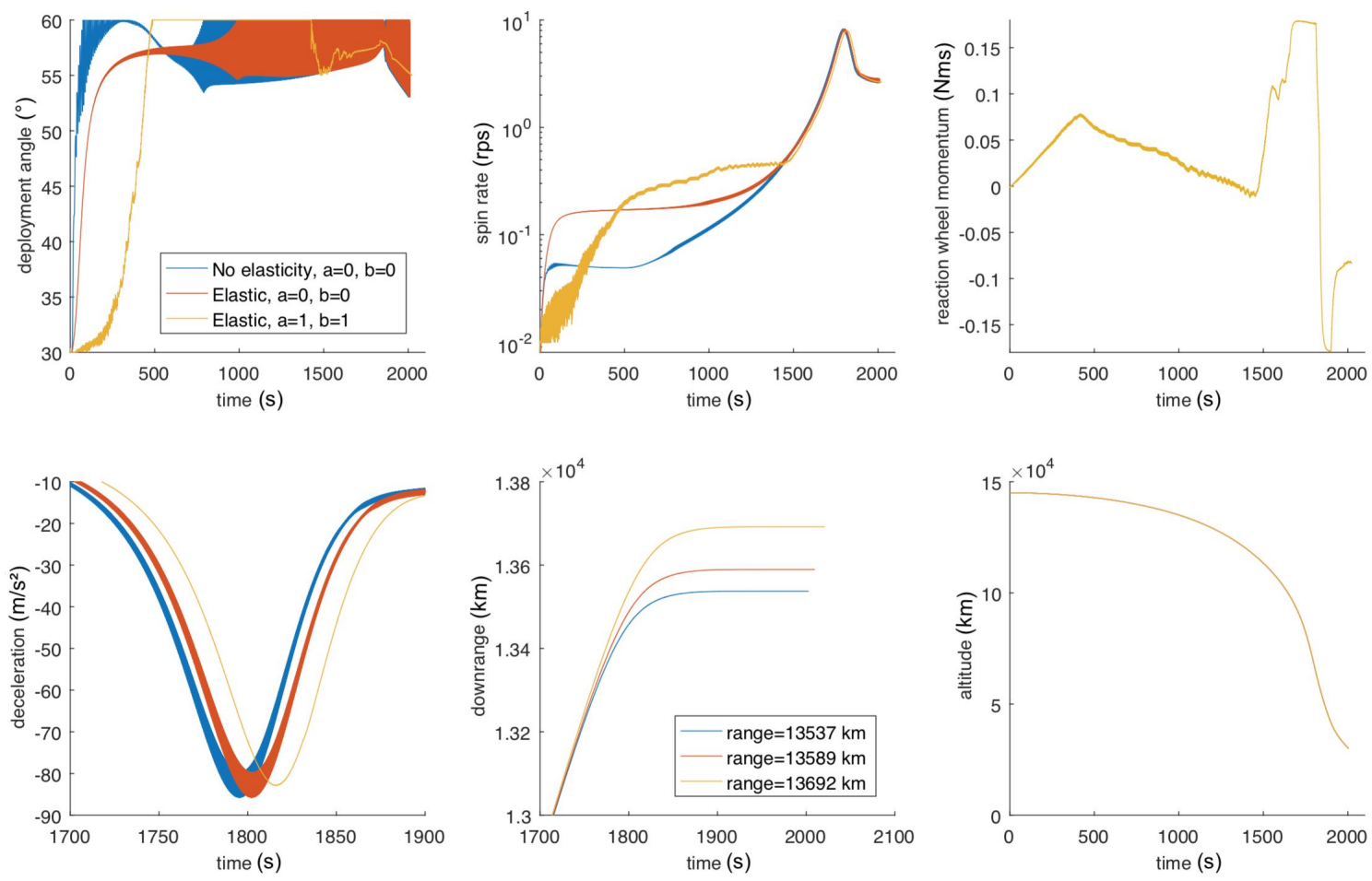

Figure 10: Trajectory simulation results without accounting for the shell's elasticity (blue), with elasticity (red), and with the controlled reaction wheel as well as elasticity (yellow), on a CubeSat-sized vehicle re-entering from $145 \mathrm{~km}$ LEO to $30 \mathrm{~km}$ altitude; it can be seen that for the uncontrolled scenarios ( $a=b=0$ according to equation (12)-(13) oscillation persists throughout the descent, which leads to fluctuation in deceleration.

momentum it can be seen that the reaction wheel not only counteracts the oscillations that has relatively high frequency $(0.1 \mathrm{~Hz} \sim 10 \mathrm{~Hz})$, but also undesirably counteracts the overall variation of spin rate during the descent. As a result, momentum saturation is inevitable regardless of downrange control gains. Therefore, the de-saturation gain scheduling (Equations (14)-(15)) is always beneficial to the performance as it conserves some momentum that is useful for oscillation suppression when the reaction wheel approaches maximum speed, rather than inducing a hardstop when the reaction wheel is saturated. With this control strategy, a near saturated wheel could "harvest" momentum (from aerodynamic roll-torque) during oscillation and tend to bring the wheel speed back to zero. This is proven effective by the simulation as oscillation is suppressed even under the near-saturated condition before the peak dynamic pressure. Meanwhile, this desaturation control also helps reducing reaction wheel power consumption as it reduces the motor speed.

\section{Downrange control}

As described in section 2, downrange manoeuvre can be achieved by manipulating the control gains $a$ and $b$ (Equations (12)-(13)), which leads to an overall spin-up or spin-down of the vehicle, thus influences the deployment angle, thereby determines the drag coefficient of the heat shield. 
According to Figure 4. downrange can be shortened by letting $a>b$ as it tends to spin-up the vehicle, and vise versa. The effect of control parameters $a$ and $b$ is already validated by Figure 8 , which shows that a higher $a / b$ ratio leads to a lower deployment angle until the reaction wheel is near-saturated. Meanwhile, due to the limited torque and momentum that the reaction wheel can output, the effect of downrange control is only eminent when the axial aerodynamic torque is low (at high altitude). Therefore, as supported by Figure 8, the controller has a greater influence on the deployment angle at $150 \mathrm{~km}$ than at $65 \mathrm{~km}$.

The effect of downrange control on re-entry trajectory is shown in Figure 11 and summarised in Table 1. The setup is similar to the simulations reported in Figure 10 with the elasticity factor $M_{E}$ included. It has shown a total downrange variation of $322 \mathrm{~km}$. According to the deceleration history, oscillation suppression is also achieved, while the peak deceleration $(\sim 8 g)$ is independent of the trajectory. It should be noted that the downrange increment $(202 \mathrm{~km}$, with respect to the unbiased condition of $a=b=1)$ is higher than the decrement $(120 \mathrm{~km})$. The reason is discussed below.
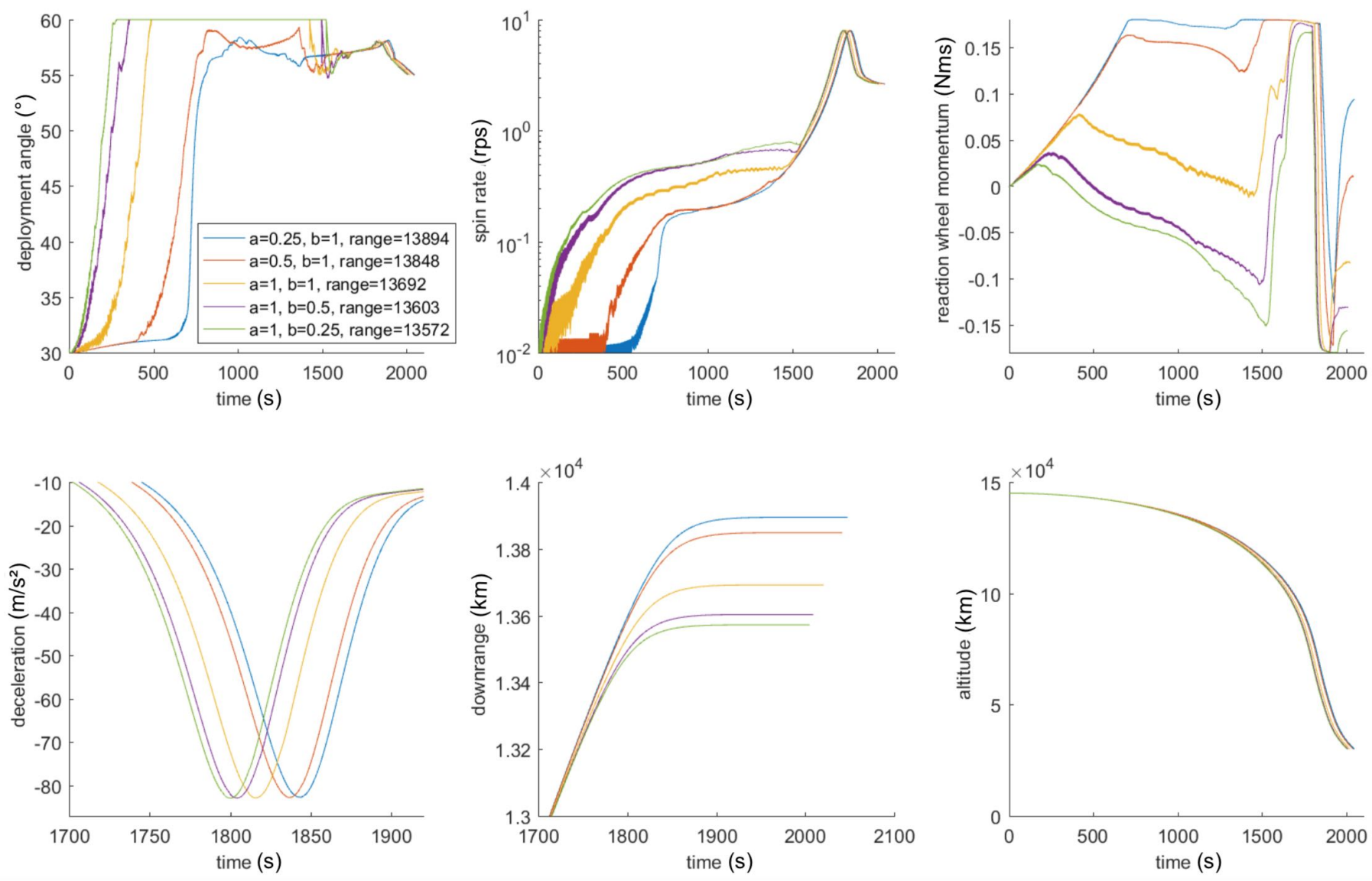

Figure 11: Trajectory simulation results on the vehicle with different downrange control gains, downrange are reported in $\mathrm{km}$, showing a downrange shift of $>300 \mathrm{~km}$ and oscillation suppressed. 
Table 1: Summary of the results from trajectory simulations of re-entry from $145 \mathrm{~km}$ altitude.

\begin{tabular}{cc}
\hline Control parameter & Downrange shift \\
\hline $\mathrm{a}=0.25, \mathrm{~b}=1$ & $202 \mathrm{~km}$ \\
$\mathrm{a}=0.5, \mathrm{~b}=1$ & $147 \mathrm{~km}$ \\
$\mathrm{a}=1, \mathrm{~b}=1$ & $0 \mathrm{~km}$ \\
$\mathrm{a}=1, \mathrm{~b}=0.5$ & $-89 \mathrm{~km}$ \\
$\mathrm{a}=1, \mathrm{~b}=0.25$ & $-120 \mathrm{~km}$ \\
\hline
\end{tabular}

It worth noticing that, according to the reaction wheel momentum history from Figure 11, the controller counteracts the increasing spin rate at the start of re-entry for $>200$ s regardless of the downrange control gains (due to the phase shift controller). This affects the capability to reduce downrange, which requires high spin rate. It can also be seen from the figure that during the downrange-reducing manoeuvres (with $a=1, b=0.5$ and 0.25 ), the reaction wheel momentum is far from saturation throughout the high altitude flight regime. Therefore, the reaction wheel's capability is currently not fully utilised, and more effective manoeuvre can potentially be achieved by more sophisticated control algorithms.

\section{Conclusions}

In the previous study, a flexible heat shield that deploys and stiffens by a self-sustaining autorotation was designed. According to the present study, the passive self-regulated centrifugal deployment leads to a limit cycle structural oscillation, which persists throughout the simulated re-entry when assuming zero structural damping. This could be a problem for sensitive payloads. The present study also showed that the heat shield is similar to a second order nonlinear system controlled by a PI controller, where the integral component is causing the oscillation.

Meanwhile, since centrifugal force is determined by the vehicle's roll rate, the heat shield shape can be easily regulated using conventional attitude control devices. Therefore, an off-the-shelf reaction wheel with a maximum power of $25 \mathrm{~W}$ and a mass of $226 \mathrm{~g}$ is proposed to regulate the autorotation (roll rate) of a CubeSat-sized vehicle and thereby achieve actively controlled deployment. The reaction wheel is controlled using a switching phase shift controller combined with gain scheduled controllers.

Specifically, the phase shift control realises oscillation suppression. The overall downrange manoeuvrability of over $300 \mathrm{~km}$ is achieved using a gain scheduled controller. Another gain scheduled controller keeps de-saturating the reaction wheel throughout the simulated re-entry. All the controls are successfully demonstrated by the numerical simulations based on Newtonian aerodynamics.

\section{References}

[1] M. Braun, P. Bruce, E. Levis, Strategies to utilize advanced heat shield technology for highpayload mars atmospheric entry missions, Acta Astronautica 136 (2017) 22-33.

[2] J. Cruz, J. Lingard, Aerodynamic decelerators for planetary exploration: past, present, and future, in: AIAA Guidance, Navigation, and Control Conference and Exhibit, AIAA paper 2006-6792, 2006. 
[3] R. D. Braun, R. M. Manning, Mars exploration entry, descent, and landing challenges, Journal of Spacecraft and Rockets 44 (2) (2007) 310-323.

[4] G. Zuppardi, R. Savino, G. Mongelluzzo, Aero-thermo-dynamic analysis of a low ballistic coefficient deployable capsule in earth re-entry, Acta Astronautica 127 (2016) 593-602.

[5] J. Andrews, K. Watry, K. Brown, Nanosat deorbit and recovery system to enable new missions, SSC11-X-3, presented at the 25th AIAA/USU Conference on Small Satellites, Logan, Utah, USA, 8-12 August, 2011.

[6] D. M. Bose, J. Shidner, R. Winski, C. Zumwalt, F. Cheatwood, S. J. Hughes, The hypersonic inflatable aerodynamic decelerator (HIAD) mission applications study, in: AIAA Aerodynamic Decelerator Systems (ADS) Conference, AIAA paper 2013-1389, 2013.

[7] J. Virgili, P. C. Roberts, N. C. Hara, Atmospheric interface reentry point targeting using aerodynamic drag control, Journal of Guidance, Control, and Dynamics 38 (3) (2015) 403413.

[8] K. Yamada, Y. Nagata, T. Abe, K. Suzuki, O. Imamura, D. Akita, Suborbital reentry demonstration of inflatable flare-type thin-membrane aeroshell using a sounding rocket, Journal of Spacecraft and Rockets 52 (1) (2014) 275-284.

[9] E. Fantino, M. Grassi, P. Pasolini, F. Causa, C. Molfese, R. Aurigemma, N. Cimminiello, D. de la Torre, P. Dell'Aversana, F. Esposito, et al., The small mars system, Acta Astronautica 137 (2017) 168-181.

[10] I. G. Clark, A. L. Hutchings, C. L. Tanner, R. D. Braun, Supersonic inflatable aerodynamic decelerators for use on future robotic missions to mars, in: Aerospace Conference, 2008 IEEE, IEEE, 2008, pp. 1-17.

[11] B. P. Smith, C. L. Tanner, M. Mahzari, I. G. Clark, R. D. Braun, F. M. Cheatwood, A historical review of inflatable aerodynamic decelerator technology development, in: Aerospace Conference, 2010 IEEE, IEEE, 2010, pp. 1-18.

[12] H. Bohon, M. Mikulas, M, Development status of attached inflatable decelerators., Journal of Spacecraft and Rockets 6 (6) (1969) 654-660.

[13] D. Litton, D. Bose, F. Cheatwood, S. Hughes, H. Wright, M. Lindell, S. Derry, A. Olds, Inflatable re-entry vehicle experiment IRVE-4 overview, in: 21st AIAA Aerodynamic Decelerator Systems Technology Conference and Seminar, AIAA paper 2011-2580, 2011.

[14] A. Mastropietro, J. Kempenaar, M. Redmond, M. Pauken, W. Ancarrow, First test flight thermal performance of the low density supersonic decelerator (LDSD) supersonic flight dynamics test (SFDT) vehicle, in: 45th International Conference on Environmental Systems, 2015.

[15] C. Tanner, J. Cruz, R. Braun, Structural verification and modeling of a tension cone inflatable aerodynamic decelerator, in: 51st AIAA/ASME/ASCE/AHS/ASC Structures, Structural Dynamics, and Materials Conference 18th AIAA/ASME/AHS Adaptive Structures Conference 12th, AIAA paper 2010-2830, 2010. 
[16] D. Wilde, S. Walther, K. Pitchadze, S. Alexsaschkin, D. Vennemann, L. Marraffa, Flight test and ISS application of the inflatable reentry and descent technology (IRDT), Acta Astronautica $51(1-9)(2002) 83-88$.

[17] V. Carandente, R. Savino, New concepts of deployable de-orbit and re-entry systems for cubesat miniaturized satellites, Recent Patents on Engineering 8 (1) (2014) 2-12.

[18] D. L. Akin, The parashield entry vehicle concept-basic theory and flight test development, in: 4th AIAA/USU Small Satellite Conference, Logan, UT, Aug. 27-30, 1990, Proceedings. (A91-27376 10-18). Logan, Vol. 1, 1990.

[19] E. Venkatapathy, K. Hamm, I. Fernandez, J. Arnold, D. Kinney, B. Laub, A. Makino, M. McGuire, K. Peterson, D. Prabhu, et al., Adaptive deployable entry and placement technology (ADEPT): a feasibility study for human missions to mars, in: 21st AIAA Aerodynamic Decelerator Systems Technology Conference and Seminar, AIAA paper 2011-2608, 2011.

[20] R. Wu, P. C. Roberts, C. Soutis, C. Diver, Flexible heat shields deployed by centrifugal force, Acta Astronautica (2018) https://doi.org/10.1016/j.actaastro.2018.06.021.

[21] R. K. Johnson, F. M. Cheatwood, A. M. Calomino, S. J. Hughes, A. M. Korzun, J. M. DiNonno, M. C. Lindell, G. T. Swanson, HIAD advancements and extension of mission applications, in: International Planetary Probe Workshop; 13th; 13-17 Jun. 2016; Laurel, MD; United States, 2016.

[22] B. Harper, R. D. Braun, Asymmetrically stacked tori hypersonic inflatable aerodynamic decelerator design study for mars entry, in: AIAA Atmospheric Flight Mechanics Conference, AIAA paper 2014-1095, 2014.

[23] N. Skolnik, H. Kamezawa, L. Li, G. A. Rossman, B. Sforzo, R. D. Braun, Design of a novel hypersonic inflatable aerodynamic decelerator for mars entry, descent, and landing, in: AIAA Atmospheric Flight Mechanics Conference, AIAA paper 2017-0469, 2017.

[24] J. S. Green, B. Dunn, R. Lindberg, Morphing hypersonic inflatable aerodynamic decelerator, in: AIAA Aerodynamic Decelerator Systems (ADS) Conference, AIAA paper 2013-1256, 2013.

[25] P. Wercinski, Adaptable deployable entry and placement technology (ADEPT), Oral/Visual Presentation ARC-E-DAA-TN31961, NASA.

[26] K. McManus, T. Poinsot, S. Candel, A review of active control of combustion instabilities, Progress in Energy and Combustion science 19 (1) (1993) 1-29.

[27] M. L. Webber, Phase shift control: Application and performance limitations with respect to thermoacoustic instabilities, Ph.D. thesis, Virginia Tech (2003).

[28] J. Hathout, M. Fleifil, A. Annaswamy, A. Ghoniem, J. Hathout, M. Fleifil, A. Annaswamy, A. Ghoniem, Why do secondary peaks occur in experimental active controllers of thermoacoustic instability?, in: 33rd Joint Propulsion Conference and Exhibit, 1997, p. 2834.

[29] W. Saunders, M. Vaudrey, B. Eisenhower, U. Vandsburger, C. Fannin, Perspectives on linear compensator designs for active combustion control, in: 37th Aerospace Sciences Meeting and Exhibit, 1999, p. 717. 
[30] Microsatellite reaction wheels (rw3-0.060), http://www.sinclairinterplanetary.com/ reactionwheels, accessed: 2018-05-02. 